\title{
OPEN Unequal on-site interaction effects in the one-dimensional electron system at quarter filling
}

\author{
Hanqin Ding ${ }^{1,2 \bowtie}$, Yan Xu $^{1} \&$ Weiting Chen ${ }^{1}$
}

The one-dimensional antiferromagnetic correlated electron system described by the unusual $t-U-J$ model with alternating on-site interactions at odd $\left(U_{o}\right)$ and even $\left(U_{e}\right)$ sites is studied analytically. At weak coupling, the use of bosonization and renormalization-group techniques helps to obtain groundstate phase diagram. At quarter filling, the unequal on-site repulsion $\left(U_{e} \neq U_{o}\right)$ causes the occurrence of umklapp processes and the generation of a charge excitation gap. Contrary to the usual case $\left(U_{e}=U_{o}\right)$, the system is not metallic but insulating. For $U_{e}+U_{0}<2 J$, the system is in a spin-gapped phase with charge-density-wave (CDW) instability; for $U_{e}+U_{0} \geq 2 J$, the system is in a spin-gapless phase characterized by the coexistence of both CDW and spin-density-wave (SDW) instabilities, where the SDW correlation dominates over the CDW one.

The discovery of high- $T_{c}$ cuprate superconductors ${ }^{1}$ has increased the interest in low-dimensional correlated systems, and particularly in the electron system characterized by interactions between the charge and spin dynamics. It is generally recognized that the crucial physics of the novel superconductor is closely related to twodimensional $\mathrm{CuO}$ planes ${ }^{2}$. By appropriately doping, the cuprates exhibit a variety of phenomena in their magnetic insulator, metal and superconductor ${ }^{3}$. The $2 \mathrm{D} t-U-J$ model is a suitable model for studying a series of phase transitions in the cuprate systems. The RVB spin-liquid state ${ }^{4}$ and the gossamer superconducting state ${ }^{5}$ are proposed as necessary precursors to high-temperature superconductivity at higher doping. In two dimensions, however, it remains very challenging to solve this model exactly. By comparison, more attention has been devoted to the one-dimensional (1D) counterpart ${ }^{6-10}$. Further more, 2D systems share some common properties with the 1D case. To a certain extent, the study of $1 \mathrm{D}$ models may act as a good guide to understanding higher-dimensional systems. It is helpful to study the $1 \mathrm{D}$ physics as a starting point and then to try to apply it to higher-dimensional cases. After all, 1D models are much easier to handle than their counterparts in higher dimensions and can even be exactly solvable. In one dimension, some analytical techniques, such as bosonization and renormalizationgroup analysis ${ }^{11-14}$, are effectively used to give the correct physics of models.

The $1 \mathrm{D} t-U-J$ model, which incorporates an nearest-neighbor spin exchange, is an extension of the standard Hubbard model. As is known, the Hubbard mode ${ }^{15}$ is the archetype of correlated electron systems. In spite of its apparent simplicity, the model does exhibit that the on-site Coulomb interaction has a strong impact on the charge dynamics, leading the $1 \mathrm{D}$ electronic system into a Mott insulator at half filling. Away from the halffilled density, however, the system becomes of a conductor. Note that the $t-U-J$ model is not an extension of the $t-J$ model. Really, the $t-J$ model is viewed as a descendant of the Hubbard model in the strong-coupling limit $(U / t \rightarrow \infty)$, where the double occupancy is completely forbidden. The $t-U-J$ model is by no means the sum of the $t-J$ and Hubbard models, because it allows for a more flexible arrangement between finite $U$ and $J$ than reflected in both models. In the case of positive interactions $(U, J>0)$, the ground state of the $1 \mathrm{D} t-U-J$ model exhibits insulating phases at half filling ${ }^{6-8}$ while is in metallic phases out of half filling ${ }^{9,10}$. The occurrence of such phenomenon is directly connected with the so-called umklapp processes, which originate from the periodicity of lattice model and stand for large momentum transfers. The lattice structure spoils the completely translational invariance, reducing the momentum conservation down to a weaker condition, where the momenta need only to be conserved modulo a vector of the reciprocal lattice $(\mathbf{G})$. In one dimension, the Fermi surface is reduced to two symmetric points, and $G=2 \pi / a$, with $a$ the lattice spacing. The umklapp processes correspond exactly to a momentum transfer of one reciprocal vector, which is equal to four times Fermi momentum $\left(k_{F}\right)$ from the lattice. $k_{F}$ is determined by the electron density $v$, defined as $k_{F}=v \pi / 2 a$. Here, the umklapp scattering refers only to the first order process. 
Notice that the above-mentioned result depends on the assumption about the umklapp processes that whether in the Hubbard model itself or in its extensions, all lattices are completely equivalent, characterized by the equal on-site interaction at each site. Within this hypothesis, one gets an umklapp process where two electrons are scattered from one side of the Fermi point to the other only if $4 k_{F}=G=2 \pi / a$. The occurrence corresponds to $v=1$, which is just the half-filled case. Deviation from such a commensurate filling, the umklapp process does not take place in one dimesion ${ }^{11,16}$. In this case, it is natural to ask the question that, what happens if the assumption is released. That is to say, if the $1 \mathrm{D}$ lattices are not equivalent, accompanied by unequal on-site interactions on different lattices, whatever will become of the fate of umklapp processes away from half filling? Really, in the early 1990s, Emery reported that there are different on-site interaction between copper and oxygen sites in the $\mathrm{Cu}-\mathrm{O}$ chain of undoped $\mathrm{La}_{2} \mathrm{CuO}_{4}$ system ${ }^{17}$. Nevertheless, the less generic situation where the site equivalence of the system is destroyed due to the introduce of several types of lattices, has been intensively discussed. By settling this subject, we will here investigate a $1 \mathrm{D}$ electron system with unequivalent lattices at odd and even sites, described by an unusual 1D extended Hubbard model with alternating on-site repulsive interaction at odd and even sites. From the viewpoint of theoretical investigation, our consideration is very essential to understand complex interactions. In one dimension, the model Hamiltonian is given by

$$
\begin{aligned}
H & =-t \sum_{j \alpha}\left(c_{j+1 \alpha}^{\dagger} c_{j \alpha}+\text { h.c. }\right)+U_{o} \sum_{j \in o d d} n_{j \uparrow} n_{j \downarrow} \\
& +U_{e} \sum_{j \in \text { even }} n_{j \uparrow} n_{j \downarrow}+J \sum_{j} \mathbf{S}_{j} \mathbf{S}_{j+1} .
\end{aligned}
$$

As usual, the $c_{j \alpha}^{\dagger}\left(c_{j \alpha}\right)$ is the creation (annihilation) operator of an electron with spin $\alpha(\uparrow$ or $\downarrow$ ) on lattice site $j$, and the number operator $n_{j \alpha}=c_{j \alpha}^{\dagger} c_{j \alpha} . S_{j}$ is the spin operator on site $j$ with $S_{j}^{\beta}=\frac{1}{2} c_{j \alpha}^{\dagger} \tau_{\alpha \alpha^{\prime}}^{\beta} c_{j \alpha^{\prime}}$, where $\tau^{\beta}$ are the Pauli matrices $(\beta=x, y, z)$. $t$ represents a single-electron nearest-neighbor hopping and $J$ denotes inter-site exchange interaction. For a change, $U_{o}$ and $U_{e}$ denote on-site interaction at odd and even sites, respectively. We only consider the case for repulsive $U_{o} \neq U_{e}$ and isotropic antiferromagnetic exchange, $U_{o}, U_{e}, J>0$. To highlight the physics of non-half-filled systems, we focus on the quarter filling where the umklapp processes are absent in the usual $t-U-J$ model $\left(U_{o}=U_{e}\right)^{9}$. To use the low-energy field theory technique effectively, we concentrate our attention on the weak-coupling regime, $U_{o}, U_{e}, J \ll t$, as in other work ${ }^{6-10}$. The result shows that the unequal on-site repulsions at nonequivalent odd and even sites gives rise to the occurrence of umklapp processes at quarter filling. The charge excitation spectrum is always massive, and the system is not metallic but insulating deviating from the $U_{o}=U_{e}$ line. In the spin sector, there is a transition from gapped excitation to gapless one. The ground state phase diagram consists of two kinds of insulating phases. The spin-gapped phase is the charge density wave (CDW). The spin-gapless phase is characterized by the coexisting CDW and spin density wave (SDW) instabilities, however, the SDW correlation is logarithmically dominant with respect to the CDW correlation. Theoretically, the result provides the further understanding of quantum properties of $1 \mathrm{D}$ correlated electron systems and the occurrence of umklapp processes.

\section{Boson representation}

Bosonization ${ }^{18,19}$ is a powerful tool to analyze $1 \mathrm{D}$ fermion systems and is extensively used to investigate groundstate phases of $1 \mathrm{D}$ extended Hubbard models ${ }^{20-28}$. This approach decouples the charge and spin degrees of freedom, and some of the interacting terms in the fermionic Hamiltonian turn into free noninteracting terms in the corresponding bosonic expressions. The $1 \mathrm{D}$ low-energy physics is mainly described by these states close to left- and right-Fermi points, around which the fermionic operators are decomposed in the continuum limit as

$$
c_{j \alpha}=e^{-i k_{F} x} \psi_{L \alpha}(x)+e^{i k_{F} x} \psi_{R \alpha}(x)
$$

Here the fermion fields $\psi_{L, \alpha}(x)$ and $\psi_{R, \alpha}(x)$ describe left-moving and right-moving particles respectively, and they contain only long wavelength components which are smooth within about one lattice spacing.

In order to carry out bosonozation technique, one first needs to introduce density operators by ${ }^{28}$

$$
\rho_{r \alpha}(x)=\psi_{r \alpha}^{\dagger}(x) \psi_{r \alpha}(x)
$$

where $r=L, R$ is the charity index (left or right). Accordingly, the charge- $\left(\rho_{r}\right)$ and spin- $\left(\sigma_{r}\right)$ density operators are defined as

$$
\rho_{r}(x)=\frac{\rho_{r \uparrow}(x)+\rho_{r \downarrow}(x)}{\sqrt{2}}, \quad \sigma_{r}(x)=\frac{\rho_{r \uparrow}(x)-\rho_{r \downarrow}(x)}{\sqrt{2}} .
$$

In the case of non-half filling $(v \neq 1)$, the complete Hamiltonian Eq. (1) is rewritten as (in what follows, we set $a=1)$ 


$$
\begin{aligned}
H & =\pi v_{F} \int \mathrm{dx}\left(\rho_{\mathrm{L}}^{2}+\rho_{\mathrm{R}}^{2}+\sigma_{\mathrm{L}}^{2}+\sigma_{\mathrm{R}}^{2}-\mathrm{g}_{\rho} \rho_{\mathrm{L}} \rho_{\mathrm{R}}-\mathrm{g}_{\sigma} \sigma_{\mathrm{L}} \sigma_{\mathrm{R}}\right) \\
& +\frac{\pi v_{F}}{2} \int \mathrm{dx}\left\{\mathrm{g}_{1 \perp}\left(\psi_{\mathrm{R}, \alpha}^{\dagger} \psi_{\mathrm{L}, \alpha} \psi_{\mathrm{L},-\alpha}^{\dagger} \psi_{\mathrm{R},-\alpha}+\text { h.c. }\right)\right. \\
& \left.+g_{3 \perp}\left[\psi_{R, \alpha}^{\dagger} \psi_{L, \alpha} \psi_{R,-\alpha}^{\dagger} \psi_{L,-\alpha} e^{i\left(\frac{1}{2}-v\right) G x}+\text { h.c. }\right]\right\}
\end{aligned}
$$

where the Fermi velocity $v_{F}=2 t \sin k_{F}$. The $g_{1 \perp}$ term describes backscattering processes, regardless of the electron fillings. Significantly differently, the $g_{3 \perp}$ terms is the umklapp process, which is active at $v=1 / 2$. The origin of phase factors $e^{i\left(\frac{1}{2}-v\right) G x}$ is shown in the Appendix, which explicitly shows that in addition to the half filling $(v=1)$, the umklapp processes take place at quarter filling $(v=1 / 2)$ in the model involved.

According to bosonization scheme, the fermion operators are expressed by bosonic operators $\phi_{r \alpha}(x)^{28}$

$$
\psi_{r \alpha}(x)=\frac{U_{r \alpha}}{\sqrt{2 \pi \epsilon}} e^{i \phi_{r \alpha}(x)}
$$

with

$$
\phi_{r \alpha}(x)= \pm 2 \pi \int \rho_{r \alpha}(x) \mathrm{dx},
$$

where the upper (lower) sign corresponds to $r=L(R) . \epsilon$ is a short-distance cutoff. The Hermitian operators $U_{r \alpha}$ ensure anticommutation relations of different fermion fields ${ }^{29}$. In terms of $\phi_{r \alpha}(x)$, we define

$$
\begin{aligned}
\phi_{r \rho}(x) & =\frac{\phi_{r \uparrow}(x)+\phi_{r \downarrow}(x)}{\sqrt{2}}, \\
\phi_{r \sigma}(x) & =\frac{\phi_{r \uparrow}(x)-\phi_{r \downarrow}(x)}{\sqrt{2}} .
\end{aligned}
$$

Based on this, we further introduce a pair of canonical variables $\phi_{\mu}(x)$ and $\theta_{\mu}(x)$,

$$
\begin{gathered}
\phi_{\mu}(x)=\frac{\phi_{L \mu}+\phi_{R \mu}}{\sqrt{4 \pi}}=\sqrt{\pi} \int\left(\mu_{L}+\mu_{R}\right) \mathrm{dx}, \\
\theta_{\mu}(x)=\sqrt{\pi} \int\left(\mu_{R}-\mu_{L}\right) \mathrm{dx},
\end{gathered}
$$

which describe the charge $(\mu=\rho)$ and spin $(\mu=\sigma)$ fields, respectively, satisfying the relation

$$
\left[\phi_{\mu}(x), \theta_{\mu^{\prime}}\left(x^{\prime}\right)\right]=\frac{\pi}{2} \delta_{\mu \mu^{\prime}} \operatorname{sgn}\left(x-x^{\prime}\right)
$$

In the present work, we focus only on the quarter-filling case, at which the umklapp process is absent in the 1D standard Hubbard model. Using the expressions (6)-(11), we convert the Hamiltonian (5) into the sum of the charge and spin sectors, $H=H_{\rho}+H_{\sigma}$, with

$$
\begin{aligned}
H_{\rho} & =\frac{v_{F}}{2} \int \mathrm{dx}\left[\left(1-\frac{\mathrm{g}_{\rho}}{2}\right)\left(\partial_{\mathrm{x}} \phi_{\rho}\right)^{2}+\left(1+\frac{\mathrm{g}_{\rho}}{2}\right)\left(\partial_{\mathrm{x}} \theta_{\rho}\right)^{2}\right] \\
& +\frac{v_{F} g_{3 \perp}}{2 \pi \epsilon^{2}} \int \mathrm{dx} \cos \left(\sqrt{8 \pi} \phi_{\rho}\right), \\
H_{\sigma} & =\frac{v_{F}}{2} \int \mathrm{dx}\left[\left(1-\frac{\mathrm{g}_{\sigma}}{2}\right)\left(\partial_{\mathrm{x}} \phi_{\sigma}\right)^{2}+\left(1+\frac{\mathrm{g}_{\sigma}}{2}\right)\left(\partial_{\mathrm{x}} \theta_{\sigma}\right)^{2}\right] \\
& +\frac{v_{F} g_{1 \perp}}{2 \pi \epsilon^{2}} \int \mathrm{dx} \cos \left(\sqrt{8 \pi} \phi_{\sigma}\right) .
\end{aligned}
$$

Up to the lowest orders of interactions, the dimensionless coupling constants are calculated as

$$
\begin{gathered}
g_{\rho}=-\frac{U_{e}+U_{o}}{2 \pi v_{F}}, \\
g_{3 \perp}=\frac{U_{o}-U_{e}}{2 \pi v_{F}}, \\
g_{\sigma}=g_{1 \perp}=\frac{1}{2 \pi v_{F}}\left(U_{e}+U_{o}-2 J\right) .
\end{gathered}
$$

In the above expressions, the relation $g_{\sigma}=g_{1 \perp}$ comes directly from the spin SU(2) symmetry of the model. 


\section{Renormalization group analysis}

In addition to free terms, the bosonized Hamiltonian contains in general interacting terms, which are signed by the cosines in Eqs. (12) and (13). Their effects can be analyzed by the renormalization-group (RG) method. To be specific, if the coefficient of a cosine decreases in the RG flow, the fixed point corresponds to a trivial theory of free bosons with known properties. On the contrary, if the RG flow scales towards strong-coupling regions, the coefficient increases. At this point, the fields are trapped in a minimum of the free energy and the different states can be characterized by computing the vacuum expectation value at this minimum of the bosonic operators ${ }^{30}$. To perform the RG scheme, we define a nonuniversal parameter closely related to both fixed-point values and correlation functions as

$$
K_{\mu}=\sqrt{\frac{2+g_{\mu}}{2-g_{\mu}}}, \quad(\mu=\rho, \sigma) .
$$

In the weak-coupling case, one has $\left|g_{\mu}\right|,\left|g_{i \perp}\right| \ll 1$, and the general relation (17) implies

$$
\begin{aligned}
K_{\mu} & =1+\frac{g_{\mu}}{2}+\mathcal{O}\left(g_{\rho}^{2}\right)+\cdots \simeq 1+\frac{g_{\mu}}{2}, \\
\frac{1}{K_{\mu}} & =1-\frac{g_{\mu}}{2}+\mathcal{O}\left(g_{\rho}^{2}\right)-\cdots \simeq 1-\frac{g_{\mu}}{2} .
\end{aligned}
$$

In terms of the expressions (17)-(19), the low-energy behavior of the models (12) and (13) can be descried by the known sine-Gordon models for the charge and the spin sectors,

$$
\begin{aligned}
H_{\mu} & =\frac{v_{\mu}}{2} \int\left[K_{\mu}^{-1}\left(\partial_{x} \phi_{\mu}\right)^{2}+K_{\mu}\left(\partial_{x} \theta_{\mu}\right)^{2}\right] \mathrm{dx} \\
& +\frac{v_{\mu} m_{\mu}}{2 \pi \epsilon^{2}} \int \cos \left(\sqrt{8 \pi} \phi_{\mu}\right) \mathrm{dx} .
\end{aligned}
$$

Here $v_{\rho} m_{\rho}=v_{F} g_{3 \perp}, v_{\sigma} m_{\sigma}=v_{F} g_{1 \perp}$. The velocities of charge $(\rho)$ and spin $(\sigma)$ excitations become modified as $v_{\mu}=v_{F} / K_{\mu}$. The effects of umklapp and backward processes are thus associated with scaling behavior of the effective masses $\left(m_{\mu}\right)$, which are accomplished by the RG analysis. For this purpose, we introduce the following RG equations ${ }^{31}$

$$
\begin{gathered}
\frac{\mathrm{d} \xi_{\mu}(\mathrm{l})}{\mathrm{dl}}=-\eta_{\mu}^{2}(l), \\
\frac{\mathrm{d} \eta_{\mu}(\mathrm{l})}{\mathrm{dl}}=-\xi_{\mu}(l) \eta_{\mu}(l),
\end{gathered}
$$

with the initial conditions $\xi_{\mu}(0)=4\left(\sqrt{K_{\mu}}-1\right) \simeq g_{\mu}, \eta_{\rho}(0)=m_{\rho} \simeq g_{3 \perp}$, and $\eta_{\sigma}(0)=m_{\sigma} \simeq g_{1 \perp}$.

The RG flows, which are characterized by scaling invariant $\xi_{\mu}^{2}-\eta_{\mu}^{2}=$ const, exhibits that the system is gapless (gapped) if the bare parameters $\xi_{\mu}(0) \geq\left|\eta_{\mu}(0)\right|\left(\xi_{\mu}(0)<\left|\eta_{\mu}(0)\right|\right)$. In the gapless case, the field $\phi_{\mu}(x)$ oscillates, and the integral of the cosine in Eq. (20) vanishes averagely. With increasing scaling length, the effective mass $m_{\mu}(l) \rightarrow 0$, and the low energy behavior of the system is described by a free scalar field. In the gapped case, $m_{\mu}(l) \rightarrow \pm \infty$, and the vacuum expectation value of the field $\left\langle\phi_{\mu}\right\rangle$ is pinned to a value that minimizes the potential. Depending on the sign of the initial mass, $\left\langle\phi_{\mu}\right\rangle=\sqrt{\pi / 8}$ for $m_{\mu}>0$, and $\left\langle\phi_{\mu}\right\rangle=0$ for $m_{\mu}<0^{16}$.

In the spin sector, the $\mathrm{SU}(2)$ symmetry drives the RG flux flow only along the separatrix $\xi_{\sigma}=\eta_{\sigma}$. This causes the spin-gap transition to takes place at $g_{\sigma}=g_{1 \perp}=0$, which is equivalent to the condition

$$
U_{o}+U_{e}=2 J \text {. }
$$

For $U_{o}+U_{e} \geq 2 J$, the spin excitation is gapless, $\Delta_{\sigma}=0$. The backward processes are irrelevant, and the correlation exponent $K_{\sigma}(l \rightarrow \infty)=K_{\sigma}^{*}=1$. In the opposite region, the excitation spectrum is gapped, $\Delta_{\sigma}>0$. The backscattering processes are relevant, $K_{\sigma}^{*}=0$, and $\left\langle\phi_{\sigma}\right\rangle=0$.

As not in the spin sector, the $\mathrm{SU}(2)$ symmetry is broken in the charge sector. Furthermore, the parameters $K_{\rho}$ and $m_{\rho}$ vary independently, and the RG flux deviates from the separatrix $\xi_{\rho}(l)=\eta_{\rho}(l)$. Since $\xi_{\rho}(0)<0$ and $\eta_{\rho}(0) \neq 0$, the charge excitation is gapped. This indicates that the umklapp processes are always relevant. There is no charge-gap transition, instead, a Gaussian transition takes place at $\eta_{\rho}(0)=0$ and $\xi_{\rho}(0)<0$. This is a type of transition between the two massive phases, which correspond to the opposite fixed points, $\eta_{\rho}(l \rightarrow \infty)= \pm \infty$, and the charge gap only closes at a critical point. According to Eq. (15), the Gaussian transition point is obtained as

$$
U_{o}=U_{e} .
$$

The value of $\left\langle\phi_{\rho}\right\rangle$ is $\sqrt{\pi / 8}$ for $U_{o}>U_{e}$ and zero for $U_{o}<U_{e}$, respectively. In the two cases, we have $K_{\rho}^{*}=0$. 


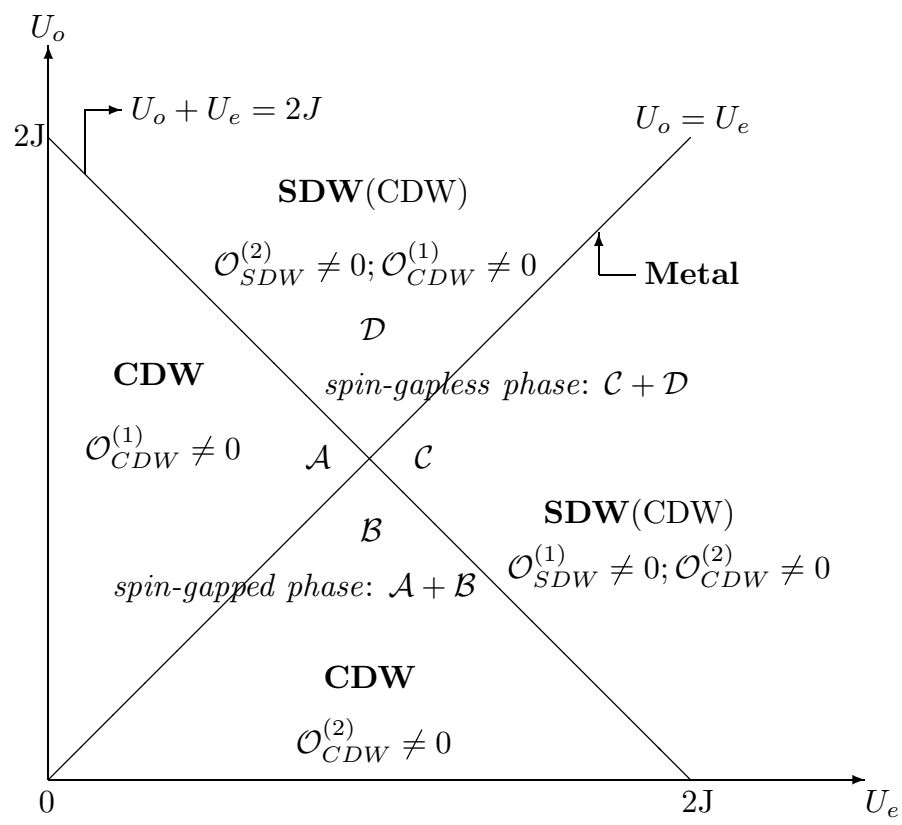

Figure 1. The weak-coupling phase diagram of $1 \mathrm{D}$ unusual $t-U-J$ model with alternating on-site repulsion at quarter filling. The subleading instability is indicated in bracket.

\section{Ground-state phase diagram}

In this sector, we determine ground-state phase diagram of the system. Because the umklapp processes are always present, the system is an insulator with a charge gap $\left(\Delta_{\rho}>0\right)$. In one dimension, the insulating phases are usually described by several types of density waves. For this purpose, we need introduce a set of order parameters. Given the non-half filling case, it is only necessary to use site-located $\mathcal{O}_{S D W}$ and $\mathcal{O}_{C D W}$, which describe the SDW and CDW instabilities, respectively. The bond-located orderings $\mathcal{O}_{B S D W}$ and $\mathcal{O}_{B C D W}$ do not need to be introduced, because they are a special feature of the half-filled band case [23]. At quarter filling, $k_{F}=\pi / 4$, and we have

$$
\begin{gathered}
\mathcal{O}_{S D W}=e^{i \pi j / 2}\left(n_{j \uparrow}-n_{j \downarrow}\right) \sim \cos \left(\frac{\pi}{2} j-\sqrt{2 \pi} \phi_{\rho}\right) \sin \sqrt{2 \pi} \phi_{\sigma}, \\
\mathcal{O}_{C D W}=e^{i \pi j / 2}\left(n_{j \uparrow}+n_{j \downarrow}\right) \sim \sin \left(\frac{\pi}{2} j-\sqrt{2 \pi} \phi_{\rho}\right) \cos \sqrt{2 \pi} \phi_{\sigma} .
\end{gathered}
$$

At odd and even sites, they are explicitly written as

$$
\begin{gathered}
\mathcal{O}_{S D W}= \begin{cases}\mathcal{O}_{S D W}^{(1)} \sim \cos \sqrt{2 \pi} \phi_{\rho} \sin \sqrt{2 \pi} \phi_{\sigma} & (j \in \text { even }) \\
\mathcal{O}_{S D W}^{(2)} \sim \sin \sqrt{2 \pi} \phi_{\rho} \sin \sqrt{2 \pi} \phi_{\sigma} & (j \in \text { odd }),\end{cases} \\
\mathcal{O}_{C D W}= \begin{cases}\mathcal{O}_{C D W}^{(1)} \sim \sin \sqrt{2 \pi} \phi_{\rho} \cos \sqrt{2 \pi} \phi_{\sigma} & (j \in \text { even }) \\
\mathcal{O}_{C D W}^{(2)} \sim \cos \sqrt{2 \pi} \phi_{\rho} \cos \sqrt{2 \pi} \phi_{\sigma} & (j \in \text { odd }) .\end{cases}
\end{gathered}
$$

The ground state phase diagram is shown in Fig. 1, in which the transition lines (23) and (24) divide the $\left(U_{o}, U_{e}\right)$ plane into four regions.

Region $\mathcal{A}: U_{e}<U_{o}<2 J-U_{e}$. The initial couplings are $g_{3 \perp}>0$ and $g_{1 \perp}<0$, so the expectation values are ordered with $\left\langle\phi_{\rho}\right\rangle=\sqrt{\pi / 8}$ and $\left\langle\phi_{\sigma}\right\rangle=0$. The fixed points correspond to $g_{3 \perp}^{*}=+\infty$ and $g_{1 \perp}^{*}=-\infty$. Except for $\mathcal{O}_{C D W}^{(1)}$, the other order parameters take zero. In the region $\mathcal{B}: U_{o}<\min \left\{U_{e} ; 2 J-U_{e}\right\}$. As in the region $\mathcal{A}$, the backward and umklapp processes are both relevant, while the negative initial couplings lead to $g_{3 \perp}^{*}=g_{1 \perp}^{*}=-\infty$, accompanied by $\left\langle\phi_{\rho}\right\rangle=\left\langle\phi_{\sigma}\right\rangle=0$. Apparently, only the order parameter $\mathcal{O}_{C D W}^{(2)}$ takes nonzero. Consequently, the system is an insulator with fully gapped CDW instability in the regions of both $\mathcal{A}$ and $\mathcal{B}$.

Region $\mathcal{C}: 2 J-U_{e}<U_{0}<U_{e}$. The backward term is marginally irrelevant, with the fixed point $g_{1 \perp}^{*}=0$, and the spin sector is gapless, with the correlation exponent $K_{\sigma}^{*}=1$. In the gapped charge sector, $g_{3 \perp}^{*}=-\infty$, and $\left\langle\phi_{\rho}\right\rangle=0$. At the same time, the spin field $\phi_{s}$ is fluctuating, not to be locked at a fixed value. The values of both $\mathcal{O}_{C D W}^{(1)}$ and $\mathcal{O}_{S D W}^{(2)}$ are equal to zero, however, $\mathcal{O}_{S D W}^{(1)}$ and $\mathcal{O}_{C D W}^{(2)}$ take nonzero values. In the region $\mathcal{D}:$ $U_{o}>\max \left\{U_{e} ; 2 J-U_{e}\right\}$. Depending on the RG analysis, we obtain $g_{3 \perp}^{*}=+\infty, g_{1 \perp}^{*}=0$, and $\left\langle\phi_{\rho}\right\rangle=\sqrt{\pi / 8}$. As a result, $\mathcal{O}_{S D W}^{(1)}=\mathcal{O}_{C D W}^{(2)}=0$. On the contrary, $\mathcal{O}_{C D W}^{(1)}$ and $\mathcal{O}_{S D W}^{(2)}$ take nonzero values. To further determine dominant instabilities, we need compute the corresponding correlation function $(C)$, which is defined at a large distance as $C_{i}(x)=\left\langle\mathcal{O}_{i}(x) \mathcal{O}_{i}^{\dagger}(0)\right\rangle^{20}$. In these two regions, we have 


$$
\begin{gathered}
C_{S D W}(x) \propto x^{-1} \ln ^{1 / 2} x, \\
C_{C D W}(x) \propto x^{-1} \ln ^{-3 / 2} x .
\end{gathered}
$$

It is easily observed that the correlation of the CDW type decays faster than that of the SDW one at large distances, but for any distance both are nonzero. Therefore, the SDW and CDW instabilities coexist in the $\mathcal{C}$ and $\mathcal{D}$ regions. However, due to the weakly logarithmical corrections, the SDW correlation dominates over the CDW one in the spin-gapless insulating phase. In fact, the coexisting SDW and CDW phenomenon has been extensively studied in organic charge-transfer solid ${ }^{33}$.

As expected, on the boundary $U_{o}=U_{e}$, the conventional $t-U-J$ model is recovered. In this case, the umklapp processes disappear. Because of massless charge excitations, the system demonstrates metallic behavior.

\section{Summary and discussion}

To investigate the occurrence of umklapp processes away from half filling, we have studied a unusual 1D extended Hubbard model, which, in addition to the conventional inter-site Heisenberg exchange interaction, includes alternating on-site repulsive interactions on odd and even lattice sites at quarter filling. In order to analyze the model analytically, we focus on the weak-coupling case, where the bosonization and renormalization group techniques are effectively applied to the $1 \mathrm{D}$ system. We find that at quarter filling the unequal Hubbard interactions lead to the generation of umklapp processes, and the charge excitations are always gapped. This behavior is completely different from that of the usual $U_{o}=U_{e}$ case, where the umklapp processes are absent, characterized by the gapless charge excitations. As usual, the backward processes are not affected, and the spin sector is either gapped or gapless, based on the relation of $U_{o}, U_{e}$ and $J$. The spin excitation is just as in the usual extended Hubbard chain with the spin-SU(2) symmetry. The spin-gap transition divides the phase diagram into two different insulating phases. For $U_{e}+U_{o}<2 J$, the system is in a spin-gapped phase with the CDW instability; for $U_{e}+U_{o} \geq 2 J$, the system is in a spin-gapless phase, characterized by the coexistence of CDW and SDW instabilities, but the SDW correlation dominates over the CDW one.

Moreover, the SDW and CDW states are independent of values of $U_{a}$ and $U_{b}$. According to the latticetranslation invariance of the system, the Hamiltonian is unchanged when we shift one lattice, but the $U_{o}$ and $U_{e}$ interactions interchange each other. Physically, using the periodic boundary condition, the ground state for $U_{o}>U_{e}$ and $U_{o}<U_{e}$ should be the same. This is clearly seen from the phase diagram, where only two phase are present, and they are separated by the spin-gap transition line $U_{e}+U_{o}=2 J$, which is determined by the competition between on-site interaction and spin exchange interaction. Equivalently, the phase diagram is not modified for the difference between $U_{e}$ and $U_{o}$, including any large $U_{e}-U_{o}$.

Note that our result depends on $1 \mathrm{D}$ calculations, and it cannot be generalized to the $2 \mathrm{D}$ case, e.g., high- $T_{c}$ cuprates, although unequal on-site interactions do exist there. This is because in two dimensions the umklapp processes are practically regardless of the filling provided $\left|k_{F}\right|$ is large enough, as is completely different from the $1 \mathrm{D}$ case. Furthermore, the 2D system include more complicated on-site and spin exchange interactions. Maybe, the result can be applied to the materials containing edge-sharing $\mathrm{Cu}-\mathrm{O}$ chains, where the low-energy physics is one-dimensional.

Received: 18 March 2021; Accepted: 10 May 2021

Published online: 24 May 2021

\section{References}

1. Bednorz, J. G. \& Muller, K. A. Possible high-Tc superconductivity in the Ba-La-Cu-O system. Z. Fur. Physik B 64, 189-193 (1986).

2. Anderson, P. W. Sources of quantum protection in high- $T_{c}$ superconductivity. Science 288, 480-482 (2000).

3. Batlogg, B. High Temperature Superconducitivity (Addison-Wesley, Redwood City, 1990).

4. Anderson, P. W. The resonating valence bond state in $\mathrm{La}_{2} \mathrm{CuO}_{4}$ and superconductivit. Science 235, 1196-1198 (1987).

5. Zhang, F. C. Gossamer superconductor, Mott insulator, and resonating valence bond state in correlated electron systems. Phys. Rev. Lett. 90, 207002 (2003).

6. Japaridze, G. I. \& Müller-Hartmann, E. Triplet superconductivity in a one-dimensional ferromagnetic t-J model. Phys. Rev. B 61, 9019-9027 (2000).

7. Dai, J. H., Feng, X. F., Xiang, T. \& Yu, Y. Gapped spin-liquid states in a one-dimensional Hubbard model with antiferromagnetic exchange interaction. Phys. Rev. B 70, 064518 (2004).

8. Feng, X. F., Xu, Z. X. \& Dai, J. H. Critical line of the SU(2) spin-gap transition in the one-dimensional $t-U-J$ model. J. Phys. Condens. Matter 16, 4245-4250 (2004).

9. Dziurzik, C., Japaridze, G. I., Schadschneider, A., Titvinidze, I. \& Zittartz, J. Triplet superconductivity in a 1D itinerant electron system with transverse spin anisotropy. Eur. Phys. J. B 51, 41-51 (2006).

10. Shi, X. L., Ding, H. Q. \& Zhang, J. Density wave instabilities in the one-dimensional metals. Chin, J. Phys. 59, 250 (2019).

11. Emery, V. J. Highly conducting one-dimensional solids (Plenum, New York, 1979).

12. Solyom, J. The Fermi gas model of one-dimensional conductors. Adv. Phys. 28, 201-303 (1979).

13. Voit, J. One-dimensional Fermi liquids. Rep. Prog. Phys. Rev. 57, 977 (1994).

14. Gogolin, A. O., Nersesyan, A. A. \& Tsvelik, A. M. Bosonization and Strongly Correlated Systems (Cambridge University Press, New York, 1998).

15. Hubbard, J. Electron correlations in narrow energy bands. Proc. R. Soc. London, Ser A 275, 238-257 (1963).

16. Giamarchi, T. Quantum Physics in One Dimension (Clarendon Press, Oxford, 2003).

17. Emery, V. J. Strong-coupling field theory and soliton doping in a one-dimensional copper-oxide model. Phys. Rev. Lett. 65, 1076-1079 (1990).

18. Delft, J. V. \& Schoeller, H. Bosonization for beginners. Ann. Phys. 7, 225-306 (1998).

19. Miranda, E. Introduction to bosonization. Braz. J. Phys. 33, 3-34 (2003).

20. Voit, J. Phase diagram and correlation functons of the half-filled Hubbard model in one dimension. Phys. Rev. B 45, 4027-4042 (1992). 
21. Tsuchiizu, M. \& Furusaki, A. Phase diagram of the one-dimensional extended Hubbard model at half filling. Phys. Rev. Lett. 88, 056402 (2002).

22. Aligia, A. A. \& Arrachea, L. Triplet superconductivity in quasi-one-dimensional systems. Phys. Rev. B 60, 15332-15338 (1999).

23. Japaridze, G. I., Kampf, A. P., Sekania, M., Kakashvili, P. \& Brune, Ph. $\eta$-pairing superconductivity in the Hubbard chain with pair hopping. Phys. Rev. B 65, 014518 (2001).

24. Otsuka, H. Ground states of the one-dimensional anisotropic extended Hubbard model. Phys. Rev. Lett. 84, 5572-5575 (2000).

25. Chen, G. H. \& Wu, Y. S. Deformed Hubbard operator, bosonization, and phase diagram of the one-dimensional t-J model. Phys. Rev. B 66, 155111 (2002).

26. Montorsi, A., Dolcini, F., Iotti, R. \& Rossi, F. Symmetry-protected topological phases of one-dimensional interacting fermions with spin-charge separation. Phys. Rev. B 95, 245108 (2017).

27. Ding, H. Q. \& Zhang, J. Phase transition in the one-dimensional pair-hopping model with unusual one-electron hopping. Phys. Lett. A 383, 2784-2788 (2019).

28. Montambaux, G., Heritier, M. \& Léderer, P. Band-filling and magnetic-field effects on the phase diagram of one-dimensional conductors. Phys. Rev. B 33, 7777-7781 (1986).

29. Nakamura, M. Tricritical behavior in the extended Hubbard chains. Phys. Rev. B 61, 16377-16392 (2000).

30. Tsuchiizu, M. \& Furusaki, A. Ground-state phase diagram of the one-dimensional half-filled extended Hubbard model. Phys. Rev. B 69, 035103 (2004).

31. Wiegmann, P. B. One-dimensional Fermi system and plane xy model. J. Phys. C 11, 1583-1598 (1978).

32. Nersesyan, A. A. Orbital antiferromagnetic ordering in a two-chain model of interacting fermions. Phys. Lett. A 153, 49-54 (1991).

33. Mazumdar, S., Ramasesha, S., Torsten, R. T. \& Campbell, D. K. Theory of coexisting charge and spin-density waves in (TMTTF) $\mathrm{Br},(\mathrm{TMTSF})_{2} \mathrm{PF}_{6}$, and $\alpha$-(BEDT-TTF) ${ }_{2} \mathrm{MHg}(\mathrm{SCN})_{4}$. Phys. Rev. Lett. 82, 1522-1525 (1999) (and references therein).

\section{Acknowledgements}

This work was supported by the National Natural Science Foundation of China (12064043 and 11864039).

\section{Author contributions}

X.Y. wrote the main manuscript text. C.W.T. wrote the Appendix. D.H.Q. constructed the model. All authors reviewed the manuscript.

\section{Competing interests}

The authors declare no competing interests.

\section{Additional information}

Correspondence and requests for materials should be addressed to H.D.

Reprints and permissions information is available at www.nature.com/reprints.

Publisher's note Springer Nature remains neutral with regard to jurisdictional claims in published maps and institutional affiliations.

(c) (i) Open Access This article is licensed under a Creative Commons Attribution 4.0 International License, which permits use, sharing, adaptation, distribution and reproduction in any medium or format, as long as you give appropriate credit to the original author(s) and the source, provide a link to the Creative Commons licence, and indicate if changes were made. The images or other third party material in this article are included in the article's Creative Commons licence, unless indicated otherwise in a credit line to the material. If material is not included in the article's Creative Commons licence and your intended use is not permitted by statutory regulation or exceeds the permitted use, you will need to obtain permission directly from the copyright holder. To view a copy of this licence, visit http://creativecommons.org/licenses/by/4.0/.

(C) The Author(s) 2021 